Supporting Information

\title{
High-Efficiency Superheated Steam Generation for Portable Sterilization under Ambient Pressure and Low Solar Flux
}

Chao Chang, Peng Tao*, Jiale Xu, Benwei Fu, Chengyi Song, Jianbo Wu, Wen

Shang, and Tao Deng*

State Key Laboratory of Metal Matrix Composites, School of Materials Science and Engineering,

Shanghai Jiao Tong University, Shanghai, 200240, China

*Email: taopeng@sjitu.edu.cn; dengtao@sjtu.edu.cn 


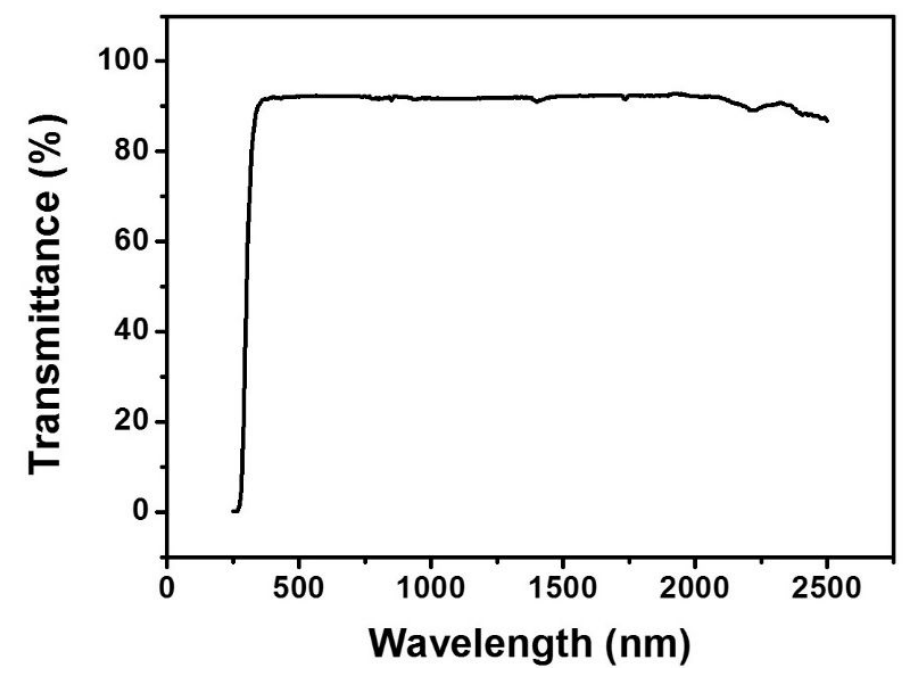

Figure S1. Transmittance spectrum of the outer glass for the solar vacuum tube.

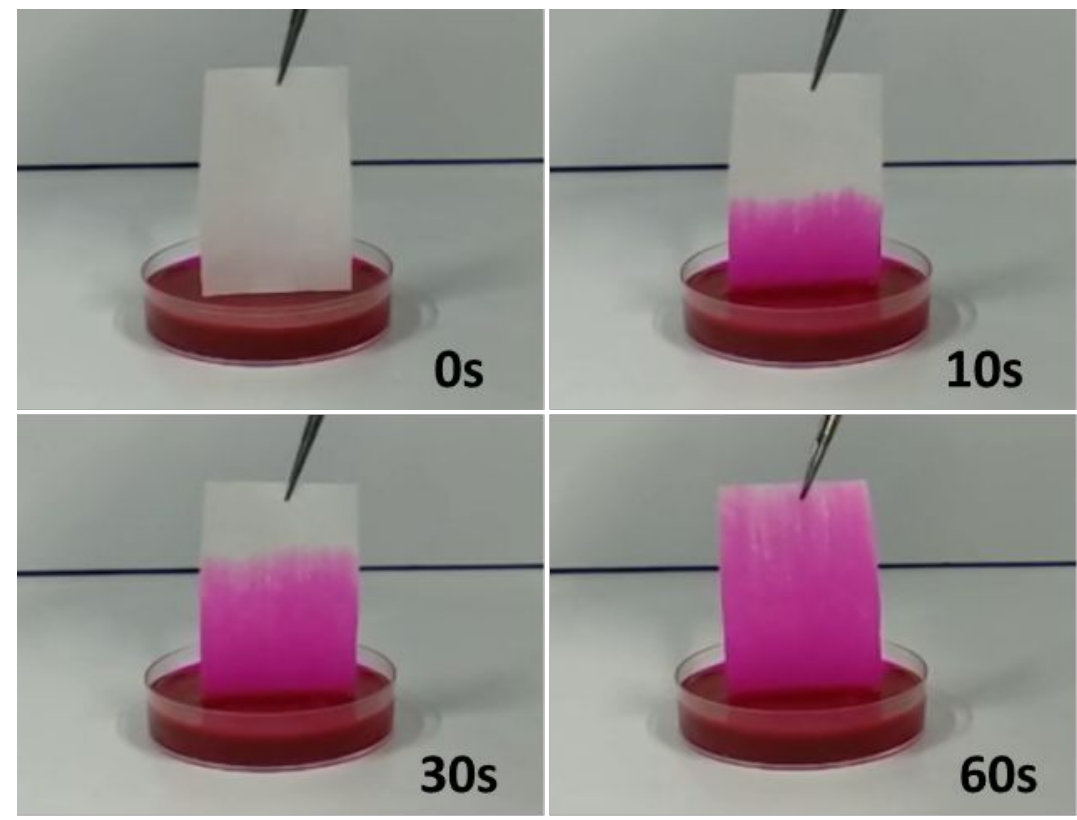

Figure S2. Wicking capacity test of the air-laid paper. 

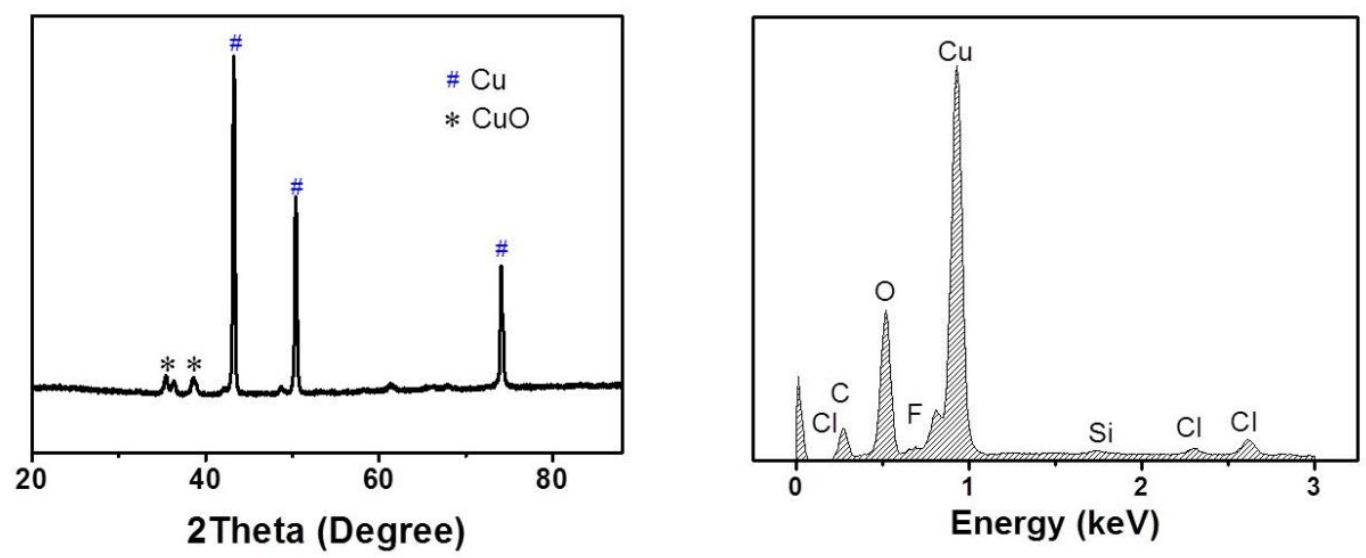

Figure S3. XRD spectrum (left) of treated hydrophilic and EDS analysis (right) of the hydrophobic copper mesh treated with fluorosilane.
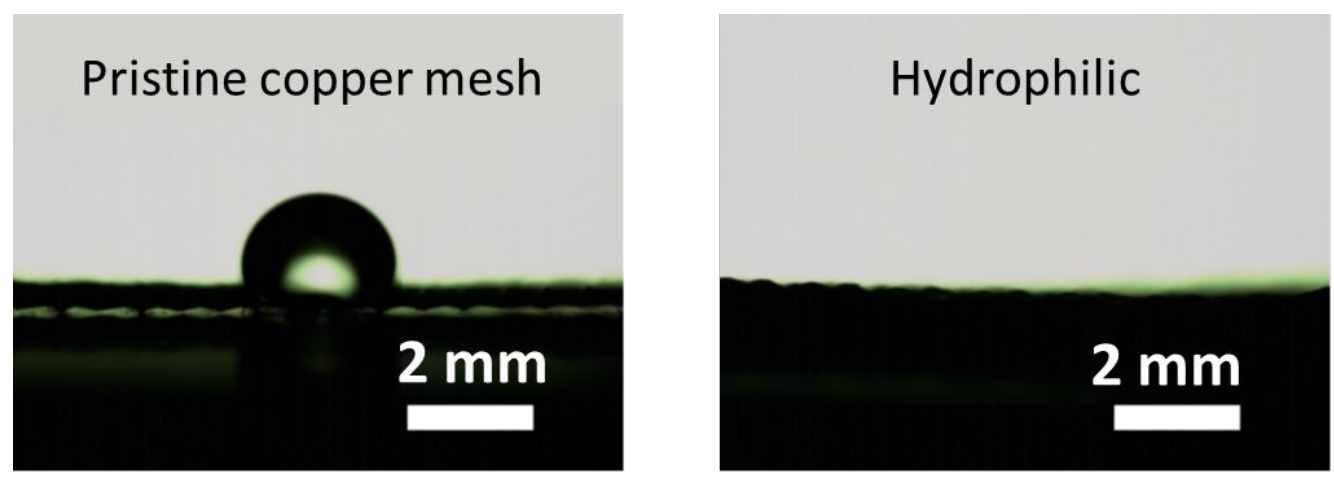

Figure S4. Contact angle measurements for the pristine copper mesh (left) and hydrophilic copper mesh (right).

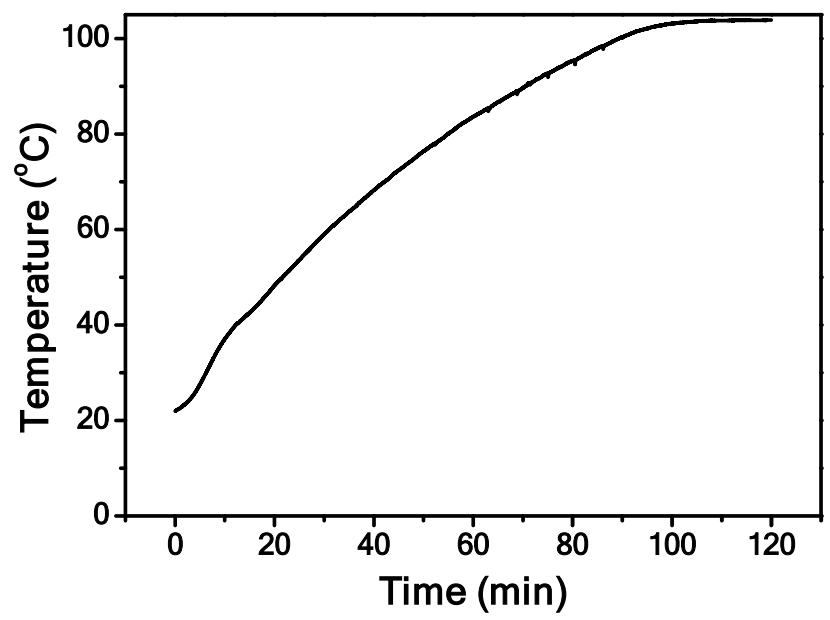

Figure S5. Temperature evolution of the non-illuminated side of the solar vacuum tube under one-sun illumination. 


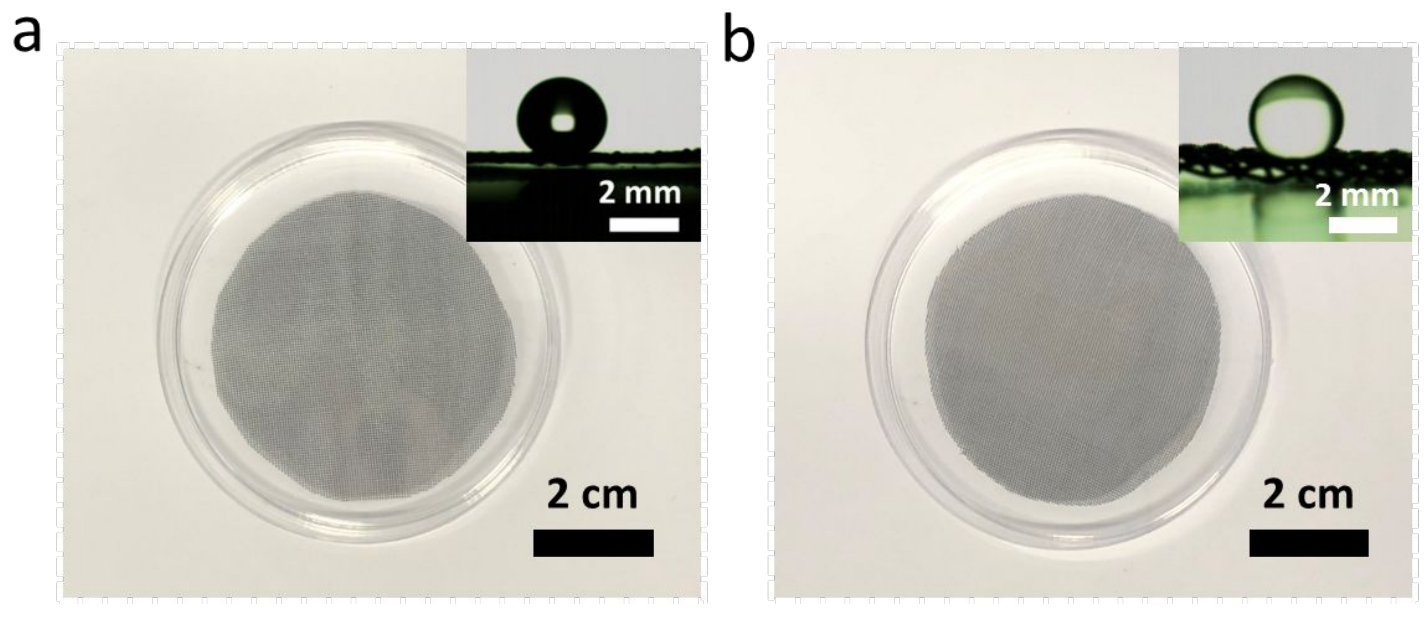

Figure S6. Comparison of the appearance and water contact angle of the hydrophobic copper mesh after continuous operation of the steam generator for 7 days: (a) before operation, (b) after operation.

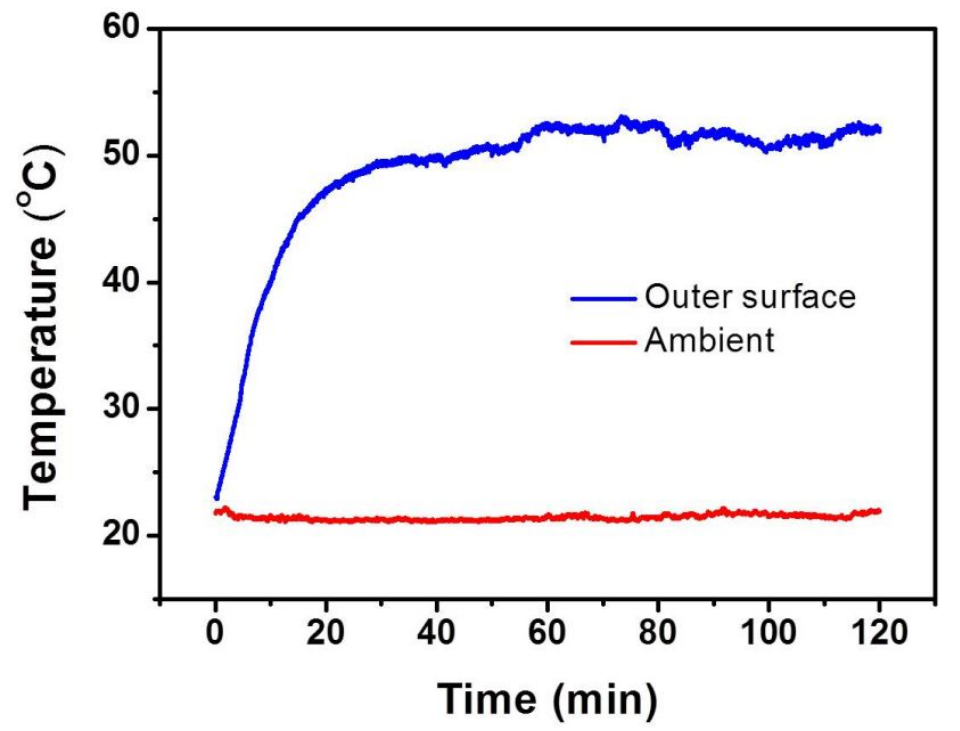

Figure S7. Temperature evolution of outer surface in the superheated steam generator with an evaporation occupation of $20 \%$. 


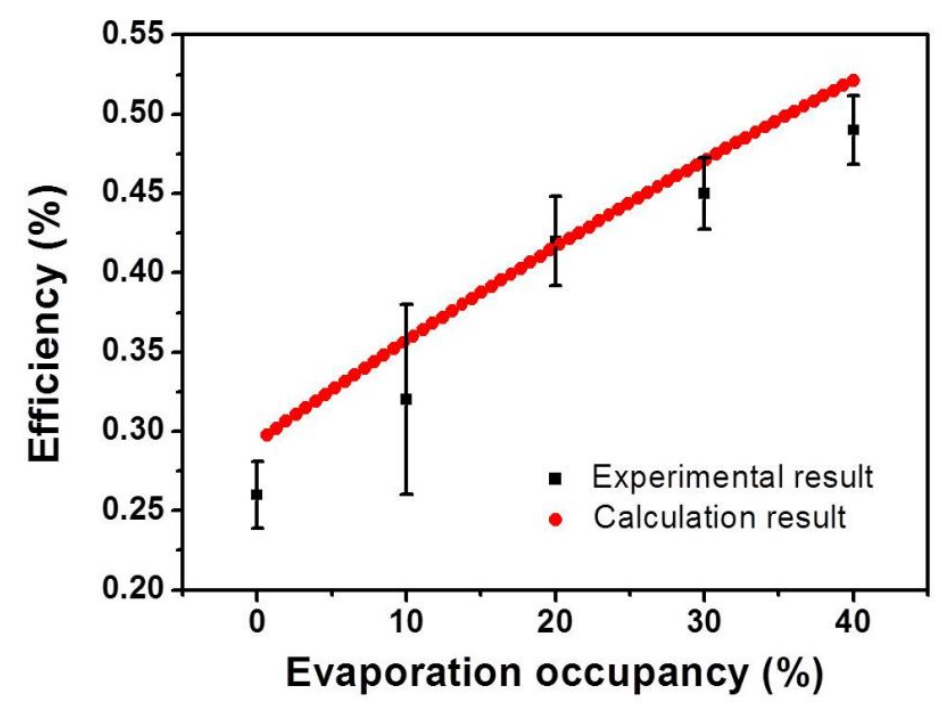

Figure S8. Evaporation efficiency of the superheated steam generator as a function of evaporation occupation.
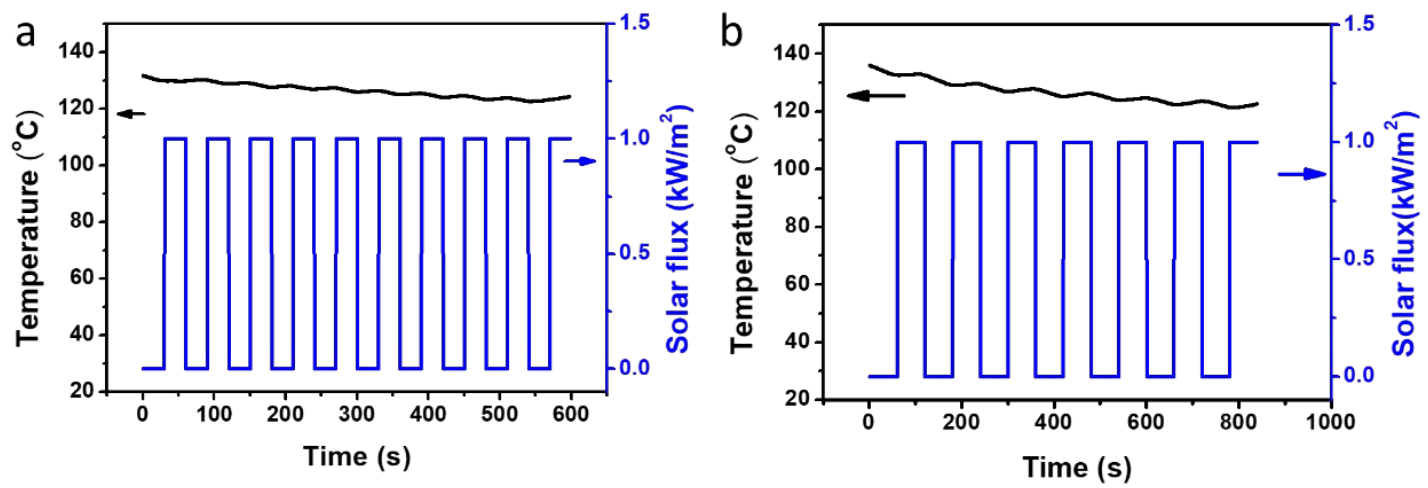

Figure S9. Steam temperature evolution under periodically switching-on and switching-off one-sun solar illumination: (a) $30 \mathrm{~s}$ (on) and $30 \mathrm{~s} \mathrm{(off),} \mathrm{and} \mathrm{(b)} 1$ min (on) and $1 \mathrm{~min}$ (off). 


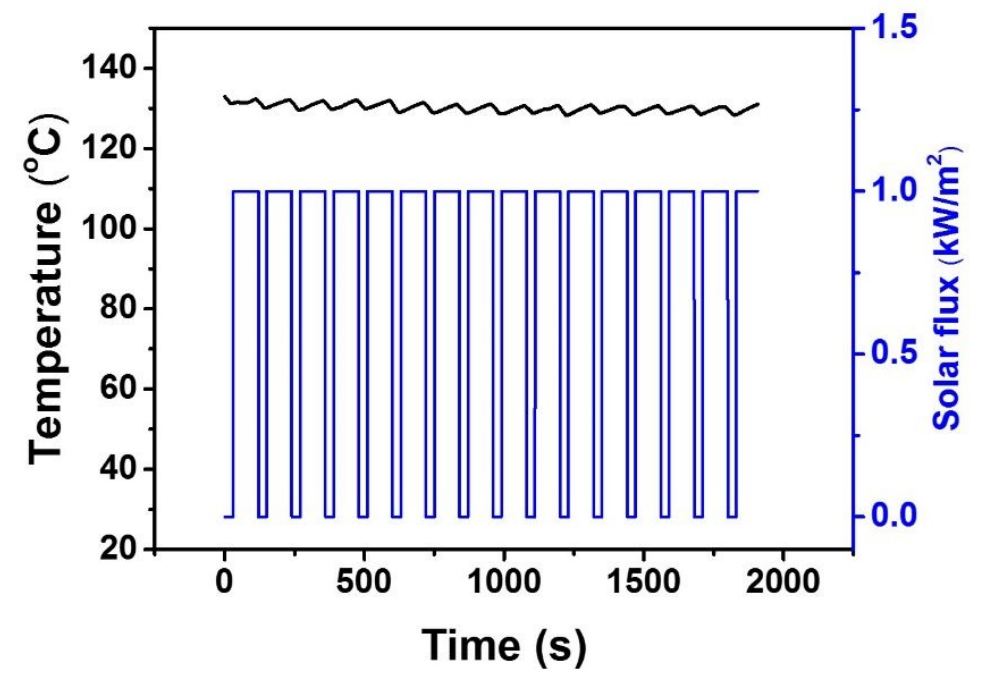

Figure S10. Steam temperature evolution under periodically switching-on and switching-off one-sun solar illumination: $90 \mathrm{~s}$ (on) and $30 \mathrm{~s}$ (off).
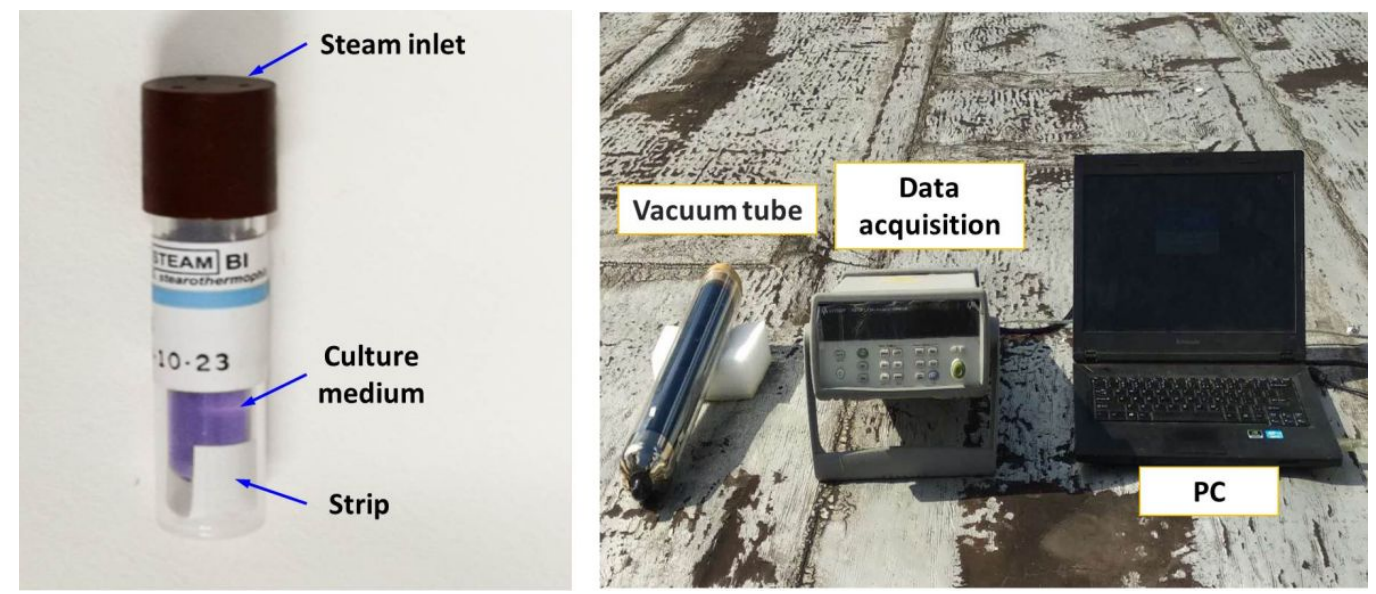

Figure S11. Schematic structure of the biological indicator (left), and the outdoor experimental setup (right). 

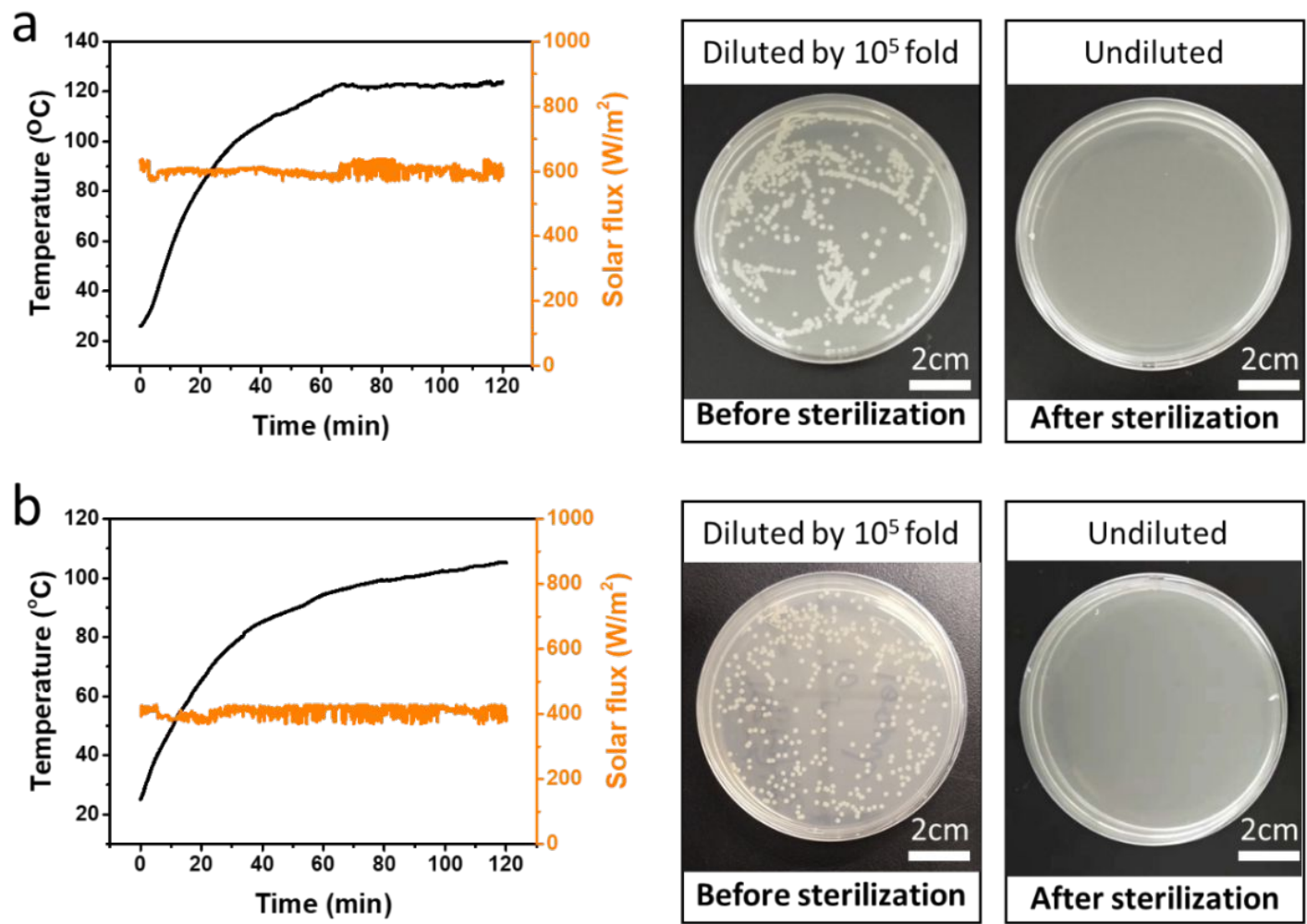

Figure S12. Steam temperature evolution of the steam generator and solar steam sterilization performance under (a) 0.6 sun and (b) 0.4 sun illumination.
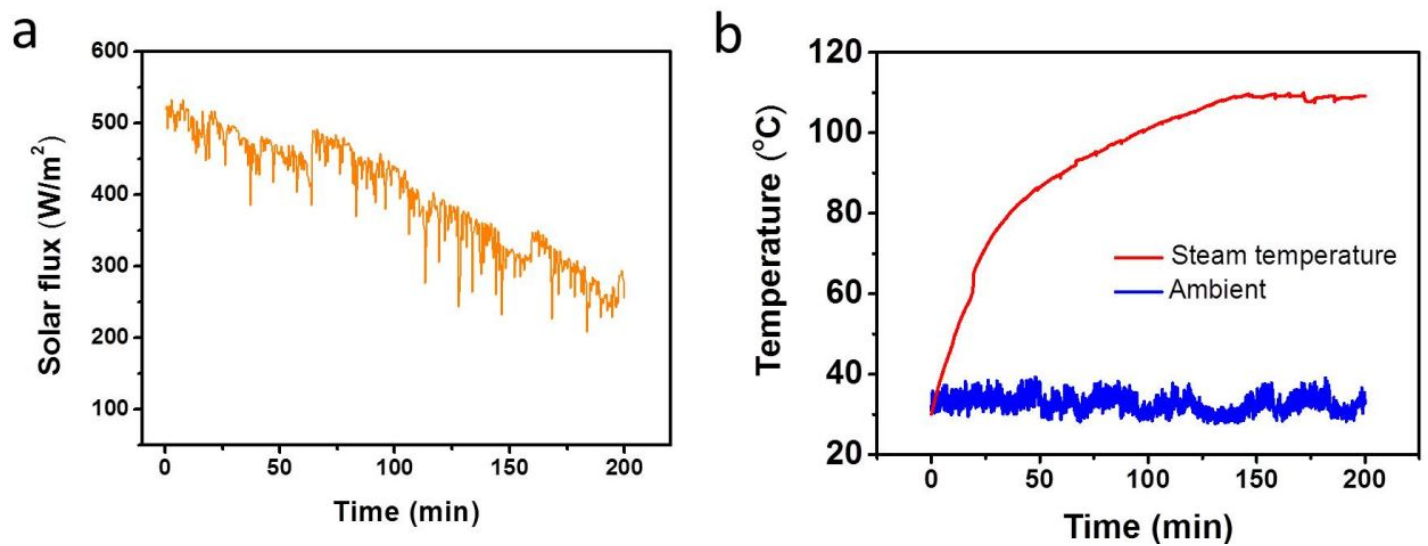

Figure S13. (a) Outdoor solar radiation on a cloudy day. (b) Steam temperature evolution over time for the superheated steam generator under the cloudy day. 


\section{Supplementary Note S1}

\section{Heat transfer analysis of the superheated steam generator}

Besides driving water evaporation $\left(q_{\text {evap }}\right)$, the incident solar energy is also lost to the environment through radiation $\left(q_{\mathrm{rad}}\right)$ and convection $\left(q_{\text {conv }}\right)$. According to energy balance principle, the energy conservation equation can be described by:

$$
q_{\mathrm{sol}} \cdot \tau_{\mathrm{glass}} \cdot \alpha_{\mathrm{abs}} \cdot A=q_{\mathrm{rad}} \cdot A^{\prime}+q_{\mathrm{conv}} \cdot A^{\prime}+q_{\mathrm{evap}}
$$

where $\tau_{\text {glass }}$ is the transmittance of the outer glass (0.92), $\alpha_{\text {abs }}$ is the absorptance of the selective absorber (0.93), $A$ is the effective solar absorption area $\left(259.05 \mathrm{~cm}^{2}\right)$, $A^{\prime}$ is the heat-dissipating area $\left(518.1 \mathrm{~cm}^{2}\right)$. The radiation heat loss $q_{\mathrm{rad}}$ and convection heat loss $q_{\text {conv }}$ can be calculated by:

$$
\begin{gathered}
q_{\text {rad }}=\varepsilon \cdot \sigma \cdot\left(T_{\mathrm{s}}^{4}-T_{\infty}{ }^{4}\right) \\
q_{\mathrm{conv}}=h \cdot\left(T_{\text {glass }}-T_{\infty}\right)
\end{gathered}
$$

where $\varepsilon$ is the emittance of the selective absorber (0.07), $\sigma$ is the Stefan-Boltzmann constant $\left(5.67 \times 10^{-8} \mathrm{~W} / \mathrm{m}^{2} \mathrm{~K}^{4}\right), \quad h$ is the convection heat transfer coefficient $\left(5 \mathrm{~W} / \mathrm{m}^{2}\right.$ $\mathrm{K}), T_{\mathrm{s}}, T_{\text {glass }}$ and $T_{\infty}$ are the temperature of the selective absorber, the outer glass and the ambient temperature, respectively. Based on the experimental temperatures, the convection and radiation heat losses can be calculated and then the evaporation efficiency is obtained. 